Proyecciones Journal of Mathematics

Vol. 29, No 3, pp. 209-226, December 2010.

Universidad Católica del Norte

Antofagasta - Chile

\title{
GENERALIZED ULAM-HYERS STABILITIES OF QUARTIC DERIVATIONS ON BANACH ALGEBRAS
}

\author{
M. ESHAGHI GORDJI \\ SEMNAN UNIVERSITY, IRAN \\ and \\ N. GHOBADIPOUR \\ URMIA UNIVERSITY, IRAN \\ Received: July 2010. Accepted : September 2010
}

\begin{abstract}
Let $\mathcal{A}, \mathcal{B}$ be two rings. A mapping $\delta: \mathcal{A} \rightarrow \mathcal{B}$ is called quartic derivation, if $\delta$ is a quartic function satisfies $\delta(a b)=a^{4} \delta(b)+\delta(a) b^{4}$ for all $a, b \in \mathcal{A}$. The main purpose of this paper to prove the generalized Hyers-Ulam-Rassias stability of the quartic derivations on Banach algebras.
\end{abstract}

2000 Mathematics Subject Classification : Primary 39B52, Secondary 39B82.

Keywords : Banach algebra; quartic functional equation; quartic derivation; Hyer-Ulam-Rassias stability. 


\section{Introduction}

The study of stability problems as just mentioned originated from a famous talk given by S.M. Ulam [65] in 1940: Under what condition does there exists a homomorphism near an approximate homomorphism? In 1941, D. H. Hyers [28] gave the first affirmative answer to the question of Ulam for Banach spaces. Let $f: E \longrightarrow E^{\prime}$ be a mapping between Banach spaces such that

$$
\|f(x+y)-f(x)-f(y)\| \leq \delta
$$

for all $x, y \in E$, and for some $\delta>0$. Then there exists a unique additive mapping $T: E \longrightarrow E^{\prime}$ such that

$$
\|f(x)-T(x)\| \leq \delta
$$

for all $x \in E$. Moreover if $f(t x)$ is continuous in $t \in \mathbf{R}$ for each fixed $x \in E$, then $T$ is linear. Finally in 1978, Th. M. Rassias [60] proved the following theorem.

Theorem 1.1. Let $f: E \longrightarrow E^{\prime}$ be a mapping from a normed vector space $E$ into a Banach space $E^{\prime}$ subject to the inequality

$$
\|f(x+y)-f(x)-f(y)\| \leq \epsilon\left(\|x\|^{p}+\|y\|^{p}\right)
$$

for all $x, y \in E$, where $\epsilon$ and $p$ are constants with $\epsilon>0$ and $p<1$. Then there exists a unique additive mapping $T: E \longrightarrow E^{\prime}$ such that

$$
\|f(x)-T(x)\| \leq \frac{2 \epsilon}{2-2^{p}}\|x\|^{p}
$$

for all $x \in E$. If $p<0$ then inequality (1.1) holds for all $x, y \neq 0$, and (1.2) for $x \neq 0$. Also, if the function $t \mapsto f(t x)$ from $\mathbf{R}$ into $E^{\prime}$ is continuous in real $t$ for each fixed $x \in E$, then $T$ is linear.

In 1991, Z. Gajda [20] answered the question for the case $p>1$, which was raised by Rassias. This new concept is known as Hyers-Ulam-Rassias stability of functional equations.

In 1982-1994, J.M. Rassias (see [46]-[53]) solved the Ulam problem for different mappings and for many Euler-Lagrange type quadratic mappings, by involving a product of different powers of norms. In addition, J.M. Rassias considered the mixed product-sum of powers of norms control function 
[61]. This concept is known as Ulam-Gavrua-Rassias stability of functional equations. For more details about the results concerning such problems and mixed product-sum stability (JMRassias Stability) the reader is referred to $[1,5,6,7,8,17,19,22,24,25,26,27,30,32,34,36,37,43,45,54,55]$ and [56].

In 1994, a generalization of the Rassias, theorem was obtained by Găvruta as follows [21] (see also [23], [31]).

Suppose $(\mathrm{G},+)$ is an abelian group, $E$ is a Banach space, and that the so-called admissible control function $\varphi: G \times G \rightarrow \mathbf{R}$ satisfies

$$
\tilde{\varphi}(x, y):=2^{-1} \sum_{n=0}^{\infty} 2^{-n} \varphi\left(2^{n} x, 2^{n} y\right)<\infty
$$

for all $x, y \in G$. If $f: G \rightarrow E$ is a mapping with

$$
\|f(x+y)-f(x)-f(y)\| \leq \varphi(x, y)
$$

for all $x, y \in G$, then there exists a unique mapping $T: G \rightarrow E$ such that $T(x+y)=T(x)+T(y)$ and $\|f(x)-T(x)\| \leq \tilde{\varphi}(x, x)$ for all $x, y \in G$.

In [40], Won-Gil Park and Jea Hyeong Bae, considered the following functional equation:

$$
f(2 x+y)+f(2 x-y)=4(f(x+y)+f(x-y))+24 f(x)-6 f(y) .
$$

In fact they proved that a function $f$ between real vector spaces $\mathrm{X}$ and $\mathrm{Y}$ is a solution of (1.3) if and only if there exists a unique symmetric multiadditive function $B: X \times X \times X \times X \longrightarrow Y$ such that $f(x)=B(x, x, x, x)$ for all $x \in X$. It is easy to show that the function $f(x)=x^{4}$ satisfies the functional equation (1.3), which is called a quartic functional equation and every solution of the quartic functional equation is said to be a quartic function (see also [2]).

Let $\mathcal{A}$ be an algebra over the real or complex field $\mathbf{F}$ and $\mathcal{X}$ a left $\mathcal{A}$-module (respectively $\mathcal{A}$-bimodule). An additive map $\delta: \mathcal{A} \rightarrow \mathcal{X}$ said to be a module left derivation (respectively module derivation) if $\delta(x y)=x . \delta(y)+y . \delta(x)$ (respectively $\delta(x y)=x . \delta(y)+\delta(x) . y$ ) holds for all $x, y \in \mathcal{A}$ where . denotes the module multiplication on $\mathcal{X}$. Since $\mathcal{A}$ is a left $\mathcal{A}$-module (respectively $\mathcal{A}$ bimodule) with the product of $\mathcal{A}$ giving the module multiplication (respectively two module multiplications), the module left derivation (respectively module derivation) $\delta: \mathcal{A} \rightarrow \mathcal{A}$ is said to be a ring left derivation (respectively ring derivation) on $\mathcal{A}$. Furthermore, if the identity $\delta(k x)=k \delta(x)$ holds for all $k \in \mathbf{F}$ and all $x \in \mathcal{A}$, then $\delta$ is a linear left derivation (respectively linear derivation). 
Let us introduce the background of our investigation.

Recently, T. Miura et al. [35] considered the stability of ring derivations on Banach algebras: Under suitable conditions, every approximate ring derivation $f$ on a Banach algebra $\mathcal{A}$ is an exact ring derivation. In particular, if $\mathcal{A}$ is a commutative semisimple Banach algebra with the maximal ideal space without isolated points, then $f$ is identically zero. The first stability result concerning derivations between operator algebras was obtained by $\mathrm{P}$. Šemrl [62] (see also [4]-[18] and [38]-[44]).

In this paper, we investigate the generalized Hyers-Ulam-Rassias stability of quartic derivations from a Banach algebra into its Banach modules.

\section{Main result}

In this section, we assume that $\mathcal{A}$ is a commutative Banach algebra and $\mathcal{X}$ a Banach $\mathcal{A}$-module.

Definition 2.1. A mapping $\delta: \mathcal{A} \rightarrow \mathcal{X}$ is called a quartic derivation if $\delta$ is a quartic function satisfies $\delta(a b)=\delta(a) b^{4}+a^{4} \delta(b)$ for all $a, b \in \mathcal{A}$.

Example 2.2. We take

$$
\mathcal{T}=\left[\begin{array}{ccccc}
0 & \mathcal{A} & \mathcal{A} & \mathcal{A} & \mathcal{A} \\
0 & 0 & \mathcal{A} & \mathcal{A} & \mathcal{A} \\
0 & 0 & 0 & \mathcal{A} & \mathcal{A} \\
0 & 0 & 0 & 0 & \mathcal{A} \\
0 & 0 & 0 & 0 & 0
\end{array}\right]
$$

Then $\mathcal{T}$ is a Banach algebra equipped with the usual matrix-like operations and the following norm:

$$
\left\|\left[\begin{array}{ccccc}
0 & a_{1} & a_{2} & a_{3} & a_{4} \\
0 & 0 & a_{5} & a_{6} & a_{7} \\
0 & 0 & 0 & a_{8} & a_{9} \\
0 & 0 & 0 & 0 & a_{10} \\
0 & 0 & 0 & 0 & 0
\end{array}\right]\right\|=\sum_{i=1}^{10}\left\|a_{i}\right\| \quad\left(a_{i} \in \mathcal{A}\right) .
$$

It is known that

$$
\mathcal{T}^{*}=\left[\begin{array}{ccccc}
0 & \mathcal{A}^{*} & \mathcal{A}^{*} & \mathcal{A}^{*} & \mathcal{A}^{*} \\
0 & 0 & \mathcal{A}^{*} & \mathcal{A}^{*} & \mathcal{A}^{*} \\
0 & 0 & 0 & \mathcal{A}^{*} & \mathcal{A}^{*} \\
0 & 0 & 0 & 0 & \mathcal{A}^{*} \\
0 & 0 & 0 & 0 & 0
\end{array}\right]
$$


is the dual of $\mathcal{T}$ under the following norm

$$
\left\|\left[\begin{array}{ccccc}
0 & f_{1} & f_{2} & f_{3} & f_{4} \\
0 & 0 & f_{5} & f_{6} & f_{7} \\
0 & 0 & 0 & f_{8} & f_{9} \\
0 & 0 & 0 & 0 & f_{10} \\
0 & 0 & 0 & 0 & 0
\end{array}\right]\right\|=\max \left\{\left\|f_{i}\right\| ; \quad f_{i} \in \mathcal{T}^{*}(i=1,2, \ldots, 10)\right\} .
$$

Let the left module action of $\mathcal{T}$ on $\mathcal{T}^{*}$ be trivial and let the right module action of $\mathcal{T}$ on $\mathcal{T}^{*}$ is defined as follows:

$$
\begin{aligned}
& \left\langle\left[\begin{array}{ccccc}
0 & f_{1} & f_{2} & f_{3} & f_{4} \\
0 & 0 & f_{5} & f_{6} & f_{7} \\
0 & 0 & 0 & f_{8} & f_{9} \\
0 & 0 & 0 & 0 & f_{10} \\
0 & 0 & 0 & 0 & 0
\end{array}\right]\left[\begin{array}{ccccc}
0 & a_{1} & a_{2} & a_{3} & a_{4} \\
0 & 0 & a_{5} & a_{6} & a_{7} \\
0 & 0 & 0 & a_{8} & a_{9} \\
0 & 0 & 0 & 0 & a_{10} \\
0 & 0 & 0 & 0 & 0
\end{array}\right],\left[\begin{array}{ccccc}
0 & b_{1} & b_{2} & b_{3} & b_{4} \\
0 & 0 & b_{5} & b_{6} & b_{7} \\
0 & 0 & 0 & b_{8} & b_{9} \\
0 & 0 & 0 & 0 & b_{10} \\
0 & 0 & 0 & 0 & 0
\end{array}\right]\right\rangle \\
& =\sum_{i=1}^{10} f_{i}\left(a_{i} b_{i}\right)
\end{aligned}
$$

for all $f_{i} \in \mathcal{A}^{*}, a_{i}, b_{i} \in \mathcal{A}(i=1, \ldots, 10)$. Then $\mathcal{T}^{*}$ is a Banach $\mathcal{T}$-module. Let

$$
\left[\begin{array}{ccccc}
0 & f_{1} & f_{2} & f_{3} & f_{4} \\
0 & 0 & f_{5} & f_{6} & f_{7} \\
0 & 0 & 0 & f_{8} & f_{9} \\
0 & 0 & 0 & 0 & f_{10} \\
0 & 0 & 0 & 0 & 0
\end{array}\right] \in \mathcal{T}^{*}
$$

We define $\delta: \mathcal{T} \rightarrow \mathcal{T}^{*}$ by

$\delta\left(\left[\begin{array}{ccccc}0 & a_{1} & a_{2} & a_{3} & a_{4} \\ 0 & 0 & a_{5} & a_{6} & a_{7} \\ 0 & 0 & 0 & a_{8} & a_{9} \\ 0 & 0 & 0 & 0 & a_{10} \\ 0 & 0 & 0 & 0 & 0\end{array}\right]\right)=\left[\begin{array}{ccccc}0 & f_{1} & f_{2} & f_{3} & f_{4} \\ 0 & 0 & f_{5} & f_{6} & f_{7} \\ 0 & 0 & 0 & f_{8} & f_{9} \\ 0 & 0 & 0 & 0 & f_{10} \\ 0 & 0 & 0 & 0 & 0\end{array}\right]\left[\begin{array}{ccccc}0 & 0 & a_{1} a_{2} & a_{3} a_{4} & a_{5} a_{6} \\ 0 & 0 & 0 & 0 & 0 \\ 0 & 0 & 0 & 0 & 0 \\ 0 & 0 & 0 & 0 & 0 \\ 0 & 0 & 0 & 0 & 0\end{array}\right]$.

Then we can see that $\delta$ is a quartic derivation from $\mathcal{T}$ into $\mathcal{T}^{*}$.

Now, we investigate the generalized Hyers-Ulam-Rassias stability of quartic derivations from $\mathcal{A}$ into $\mathcal{X}$. For convenience, we use the following abbreviation for a given mapping $f: \mathcal{A} \rightarrow \mathcal{X}$;

$D_{f}(x, y)=f(2 x+y)+f(2 x-y)-4[f(x+y)+f(x-y)]-24 f(x)+6 f(y)$ 
for all $x, y \in \mathcal{A}$.

Theorem 2.3. Let $f: \mathcal{A} \rightarrow \mathcal{X}$ with $f(0)=0$ be a mapping for which there exists function $\varphi: \mathcal{A} \times \mathcal{A} \times \mathcal{A} \times \mathcal{A} \rightarrow[0, \infty)$ such that

$$
\begin{gathered}
\left\|D_{f}(x, y)+f(z t)-z^{4} f(t)-f(z) t^{4}\right\| \leq \varphi(x, y, z, t), \\
\tilde{\varphi}(x):=\sum_{i=0}^{\infty} \frac{1}{16^{i}} \varphi\left(2^{i} x, 0,0,0\right)<\infty, \\
\lim _{i \rightarrow \infty} \frac{1}{16^{i}} \varphi\left(2^{i} x, 2^{i} y, 2^{i} z, 2^{i} t\right)=0
\end{gathered}
$$

for all $x, y, z, t \in \mathcal{A}$. Then there exists a unique quartic derivation $\delta: \mathcal{A} \rightarrow \mathcal{X}$ such that

$$
\|\delta(x)-f(x)\| \leq \frac{1}{32} \tilde{\varphi}(x)
$$

for all $x \in \mathcal{A}$.

Proof. Letting $z=t=y=0$ in (2.1), we get

$$
\left\|\frac{1}{16} f(2 x)-f(x)\right\| \leq \frac{1}{32} \varphi(x, 0,0,0)
$$

for all $x \in \mathcal{A}$. By induction, we have

$$
\left\|\frac{1}{16^{n}} f\left(2^{n} x\right)-f(x)\right\| \leq \frac{1}{32} \sum_{i=0}^{n-1} \frac{1}{16^{i}} \varphi\left(2^{i} x, 0,0,0\right)
$$

for all $x \in \mathcal{A}$. In order to show that functions $\delta_{n}(x)=\frac{1}{16^{n}} f\left(2^{n} x\right)$ form a Convergent sequence, we used Cauchy convergence criterion. In deed, replace $x$ by $2^{m} x$ in (2.6) and result divide by $16^{m}$, where $m$ is an arbitrary positive integer, we find that

$$
\left\|\frac{1}{16^{n+m}} f\left(2^{n+m} x\right)-\frac{1}{16^{m}} f\left(2^{m} x\right)\right\| \leq \frac{1}{32} \sum_{i=m}^{m+n-1} \frac{1}{16^{i}} \varphi\left(2^{i} x, 0,0,0\right)
$$

for all $x \in \mathcal{A}$. By $(2.2)$ and since $\mathcal{X}$ is complete then by $n \rightarrow \infty, \lim _{n \rightarrow \infty} \delta_{n}(x)$ exists for all $x \in \mathcal{A}$. 
Let $m=0$ and $n \rightarrow \infty$ in (2.7), we have

$$
\|\delta(x)-f(x)\| \leq \frac{1}{32} \sum_{i=0}^{\infty} \frac{1}{16^{i}} \varphi\left(2^{i} x, 0,0,0\right)=\frac{1}{32} \tilde{\varphi}(x)
$$

such that $\delta$ is defined $\delta: \mathcal{A} \rightarrow \mathcal{X}$ by $\delta(x)=\lim _{n \rightarrow \infty} \frac{1}{16^{n}} f\left(2^{n} x\right)$ for all $x \in \mathcal{A}$. Letting $z=t=0$ and replacing $x, y$ by $2^{n} x, 2^{n} y$, respectively, in the inequality (2.1), we get

$$
\left\|D_{f}\left(2^{n} x, 2^{n} y\right)\right\| \leq \varphi\left(2^{n} x, 2^{n} y, 0,0\right)
$$

for all $x, y \in \mathcal{A}$, that is,

$$
\left\|\frac{1}{16^{n}} D_{f}\left(2^{n} x, 2^{n} y\right)\right\| \leq \frac{1}{16^{n}} \varphi\left(2^{n} x, 2^{n} y, 0,0\right)
$$

for all $x, y \in \mathcal{A}$. Passing the limit $n \rightarrow \infty$, we have

$$
D_{\delta}(x, y)=0
$$

for all $x, y \in \mathcal{A}$. Hence $\delta$ is a quartic functional equation. On the other hand, letting $x=y=0$ and replacing $z, t$ by $2^{n} z, 2^{n} t$, respectively, in (2.1), we obtain

$$
\left\|f\left(2^{2 n} z t\right)-16^{n} z f\left(2^{n} t\right)-f\left(2^{n} z\right) 16^{n} t\right\| \leq \varphi\left(0,0,2^{n} z, 2^{n} t\right)
$$

for all $z, t \in \mathcal{A}$. Hence

$$
\left\|\frac{1}{16^{2 n}} f\left(2^{2 n} z t\right)-\frac{1}{16^{n}} z f\left(2^{n} t\right)-f\left(2^{n} z\right) \frac{1}{16^{n}} t\right\| \leq \frac{1}{16^{2 n}} \varphi\left(0,0,2^{n} z, 2^{n} t\right)
$$

for all $z, t \in \mathcal{A}$. Passing the limit $n \rightarrow \infty$, we obtain

$$
\delta(z t)=z^{4} \delta(t)+\delta(z) t^{4}
$$

for all $z, t \in \mathcal{A}$.

Now, suppose there exists a function $\delta^{\prime}: \mathcal{A} \rightarrow \mathcal{X}$ with

$$
D_{\delta^{\prime}}(x, y)=0
$$

for all $x, y \in \mathcal{A}$ and

$$
\left\|\delta^{\prime}(x)-f(x)\right\| \leq \frac{1}{32} \tilde{\varphi}(x)
$$

for all $x \in \mathcal{A}$. 
We have $\left\|\delta(x)-\delta^{\prime}(x)\right\|=\frac{1}{16^{n}}\left\|\delta\left(2^{n} x\right)-\delta^{\prime}\left(2^{n} x\right)\right\|=\frac{1}{16^{n}}\left(\| \delta\left(2^{n} x\right)-\right.$ $\left.f\left(2^{n} x\right)\|+\| \delta^{\prime}\left(2^{n} x\right)-f\left(2^{n} x\right) \|\right) \leq \frac{1}{16} \sum_{i=n}^{\infty} \frac{1}{16^{i}} \varphi\left(2^{i} x, 0\right)$

for all $x \in \mathcal{A}$. Passing the limit $n \rightarrow \infty$, we obtain $\delta(x)=\delta^{\prime}(x)$ for all $x \in \mathcal{A}$.

Now, we establish the Ulam-Gavruta-Rassias stability of quadratic derivations as follows:

Corollary 2.4. Let $p>0, q_{j}>0,(j=1,2,3,4)$ and $\theta$ be positive real numbers with

$$
\operatorname{Max}\left\{p, \sum_{j=1}^{4} q_{j}\right\}<4 .
$$

If $f: \mathcal{A} \rightarrow \mathcal{X}$ with $f(0)=0$ is a mapping such that

$$
\begin{gathered}
\left\|D_{f}(x, y)+f(z t)-z^{4} f(t)-f(z) t^{4}\right\| \\
\leq \theta\left(\|x\|^{p}+\|y\|^{p}+\|z\|^{p}+\|t\|^{p}+\|x\|^{q_{1}}\|y\|^{q_{2}}\|z\|^{q_{3}}\|t\|^{q_{4}}\right)
\end{gathered}
$$

for all $x, y, z, t \in \mathcal{A}$, then there is a unique quartic derivation $\delta: \mathcal{A} \rightarrow \mathcal{X}$ such that

$$
\|\delta(x)-f(x)\| \leq \frac{\theta}{32-2^{p+1}}\|x\|^{p}
$$

for all $x \in \mathcal{A}$.

Proof. The proof follows from Theorem 2.1 taking

$$
\varphi(x, y, z, t):=\theta\left(\|x\|^{p}+\|y\|^{p}+\|z\|^{p}+\|t\|^{p}+\|x\|^{q_{1}}\|y\|^{q_{2}}\|z\|^{q_{3}}\|t\|^{q_{4}}\right)
$$

for all $x, y, z, t \in \mathcal{A}$.

Moreover, we investigate the superstability of quartic derivations as follows:

Corollary 2.5. Let $q_{j}>0,(j=1,2,3,4)$ with $\sum_{j=1}^{4} q_{j}<4$, and $\theta$ be positive real numbers. If $f: \mathcal{A} \rightarrow \mathcal{X}$ with $f(0)=0$ is a mapping

$$
\left\|D_{f}(x, y)+f(z t)-z^{4} f(t)-f(z) t^{4}\right\| \leq \theta\left(\|x\|^{q_{1}}\|y\|^{q_{2}}\|z\|^{q_{3}}\|t\|^{q_{4}}\right)
$$

for all $x, y, z, t \in \mathcal{A}$, then $f$ is a quartic derivation. 
Proof. It follows from Theorem 2.1 by putting

$$
\varphi(x, y, z, t):=\theta\left(\|x\|^{q_{1}}\|y\|^{q_{2}}\|z\|^{q_{3}}\|t\|^{q_{4}}\right)
$$

for all $x, y, z, t \in \mathcal{A}$.

Theorem 2.6. Let $p_{1}+p_{2}<4, q_{1}+q_{2}<8$ and $\theta$ be positive real numbers. If $f: \mathcal{A} \rightarrow \mathcal{X}$ is a mapping

$$
\left\|D_{f}(x, y)+f(z t)-z^{4} f(t)-f(z) t^{4}\right\| \leq \theta\left(\|x\|^{p_{1}}\|y\|^{p_{2}}+\|z\|^{q_{1}}\|t\|^{q_{2}}\right)
$$

for all $x, y, z, t \in \mathcal{A}$, then there is a unique quartic derivation $\delta: \mathcal{A} \rightarrow \mathcal{X}$ such that

$$
\|\delta(x)-f(x)\| \leq \frac{\theta}{3^{4}-3^{p_{1}+p_{2}}}\|x\|^{p_{1}+p_{2}}
$$

for all $x \in \mathcal{A}$.

Proof. In the inequality (2.8), let $y=x=z=t=0$, then $23\|f(0)\| \leq 0$. Hence $f(0)=0$. Letting $y=z=t=0$ in (2.8), we see that $2 f(x)=2^{4} f(x)$ for all $x \in \mathcal{A}$. In the inequality (2.8), put $z=t=0$ and replace $y$ with $x$. Then we obtain

$$
\|f(3 x)-81 f(x)\| \leq \theta\|x\|^{p_{1}+p_{2}}
$$

for all $x \in \mathcal{A}$. Hence

$$
\left\|\frac{f(3 x)}{81}-f(x)\right\| \leq \frac{\theta}{81}\|x\|^{p_{1}+p_{2}}
$$

for all $x \in \mathcal{A}$. By using the induction, we can get that

$$
\left\|\frac{f\left(3^{n} x\right)}{81^{n}}-f(x)\right\| \leq \frac{\theta\|x\|^{p_{1}+p_{2}}}{81} \sum_{i=0}^{n-1} \frac{3^{i\left(p_{1}+p_{2}\right)}}{81^{i}}
$$

for all $x \in \mathcal{A}$. It follows from $p_{1}+p_{2}<4$ that the sequence $\left\{\frac{1}{81^{n}} f\left(3^{n} x\right)\right\}$ is Cauchy sequence and so it is convergent since $\mathcal{X}$ is complete. Thus we can define a function $\delta: \mathcal{A} \rightarrow \mathcal{X}$ given by

$$
\delta(x):=\lim _{n \rightarrow \infty} \frac{1}{81^{n}} f\left(3^{n} x\right)
$$

for all $x \in \mathcal{A}$. In (2.12), passing the limit $n \rightarrow \infty$, we obtain the inequality (2.9). The proof of the uniqueness of $\delta$, is similar to the proof of Theorem 2.1 . 
Theorem 2.7. Let $p_{1}+p_{2}>4, q_{1}+q_{2}>8$ and $\theta$ be positive real numbers. If $f: \mathcal{A} \rightarrow \mathcal{X}$ is a mapping satisfying (2.8), then there is a unique quartic derivation $\delta: \mathcal{A} \rightarrow \mathcal{X}$ such that

$$
\|f(x)-\delta(x)\| \leq \frac{\theta 3^{-\left(p_{1}+p_{2}\right)}}{1-3^{4-\left(p_{1}+p_{2}\right)}}\|x\|^{p_{1}+p_{2}}
$$

for all $x \in \mathcal{A}$.

Proof. It follows from (2.10) that

$$
\left\|f(x)-81 f\left(\frac{x}{3}\right)\right\| \leq \frac{\theta}{3^{p_{1}+p_{2}}}\|x\|^{p_{1}+p_{2}}
$$

for all $x \in \mathcal{X}$. By using the induction, we can get that

$$
\left\|f(x)-81^{n} f\left(\frac{x}{3^{n}}\right)\right\| \leq \frac{\theta\|x\|^{p_{1}+p_{2}}}{81} \sum_{i=1}^{n} \frac{81^{i}}{3^{i\left(p_{1}+p_{2}\right)}}
$$

for all $x \in \mathcal{A}$. It follows from $p_{1}+p_{2}>4$ that the sequence $\left.81^{n} f\left(\frac{x}{3^{n}}\right)\right\}$ is Cauchy sequence and so it is convergent since $\mathcal{X}$ is complete. Thus we can define a function $\delta: \mathcal{A} \rightarrow \mathcal{X}$ given by

$$
\delta(x):=\lim _{n \rightarrow \infty} 81^{n} f\left(\frac{x}{3^{n}}\right)
$$

for all $x \in \mathcal{A}$. The rest of the proof is similar to the proof of Theorem 2.3.

Theorem 2.8. Let $f: \mathcal{A} \rightarrow \mathcal{X}$ with $f(0)=0$ be a mapping for which there exists function $\varphi: \mathcal{A} \times \mathcal{A} \times \mathcal{A} \times \mathcal{A} \rightarrow[0, \infty)$ such that

$$
\begin{gathered}
\left\|D_{f}(x, y)+f(z t)-z^{4} f(t)-f(z) t^{4}\right\| \leq \varphi(x, y, z, t), \\
\tilde{\varphi}(x):=\sum_{i=1}^{\infty} 16^{i} \varphi\left(2^{-i} x, 0,0,0\right)<\infty \\
\lim _{i \rightarrow \infty} 16^{2 i} \varphi\left(2^{-i} x, 2^{-i} y, 2^{-i} z, 2^{-i} t\right)=0
\end{gathered}
$$

for all $x, y, z, t \in \mathcal{A}$. Then there exists a unique quartic derivation $\delta: \mathcal{A} \rightarrow \mathcal{X}$ such that

$$
\|f(x)-\delta(x)\| \leq \frac{1}{32} \tilde{\varphi}(x)
$$

for all $x \in \mathcal{A}$. 
Proof. It follows from (2.5) that

$$
\left\|f(x)-16 f\left(2^{-1} x\right)\right\| \leq 2^{-1} \varphi\left(2^{-1} x, 0,0,0\right)
$$

for all $x \in \mathcal{A}$. In (2.21), multiply the both sides by 16 and replace $x$ with $2^{-1} x$, we have

$$
\left\|16 f\left(2^{-1} x\right)-16^{2} f\left(2^{-2} x\right)\right\| \leq 2^{-1} 16 \varphi\left(2^{-2} x, 0,0,0\right)
$$

for all $x \in \mathcal{A}$. From two inequalities (2.21) and (2.22), we get

$$
\left\|f(x)-16^{2} f\left(2^{-2} x\right)\right\| \leq 2^{-1} \varphi\left(2^{-1} x, 0,0,0\right)+2^{-1} 16 \varphi\left(2^{-2} x, 0,0,0\right)
$$

for all $x \in \mathcal{A}$. Continuing this way, we get

$$
\left\|f(x)-16^{n} f\left(2^{-n} x\right)\right\| \leq \frac{1}{32} \sum_{i=1}^{n} 16^{i} \varphi\left(2^{-i} x, 0,0,0\right)
$$

for all $x \in \mathcal{A}$. For any positive integer $m$, multiply the both sides by $16^{m}$ and replace $x$ by $2^{-m} x$ in $(2.24)$, then we have

$$
\left\|16^{m} f\left(2^{-m} x\right)-16^{n+m} f\left(2^{-(n+m)} x\right)\right\| \leq \frac{1}{32} \sum_{i=1}^{n} 16^{i+m} \varphi\left(2^{-(i+m)} x, 0,0,0\right)
$$

for all $x \in \mathcal{A}$. Passing the limit $m \rightarrow \infty$, the sequence $\left\{16^{n} f\left(2^{-n} x\right)\right\}$ is a Cauchy sequence in $\mathcal{X}$. By the completeness of $\mathcal{X}$, the sequence $\left\{16^{n} f\left(2^{-n} x\right)\right\}$ converges and so we can define a function $\delta: \mathcal{A} \rightarrow \mathcal{X}$ given by

$$
\delta(x)=\lim _{n \rightarrow \infty} 16^{n} f\left(2^{-n} x\right)
$$

for all $x \in \mathcal{A}$. The rest of the proof is similar to the proof of Theorem 2.1.

Corollary 2.9. Let $p>0, q_{j}>0,(j=1,2,3,4)$ and $\theta$ be positive real numbers with

$$
\operatorname{Min}\left\{p, \sum_{j=1}^{4} q_{j}\right\}>4 .
$$

If $f: \mathcal{A} \rightarrow \mathcal{X}$ with $f(0)=0$ is a mapping such that

$$
\left\|D_{f}(x, y)+f(z t)-z^{4} f(t)-f(z) t^{4}\right\|
$$




$$
\leq \theta\left(\|x\|^{p}+\|y\|^{p}+\|z\|^{p}+\|t\|^{p}+\|x\|^{q_{1}}\|y\|^{q_{2}}\|z\|^{q_{3}}\|t\|^{q_{4}}\right)
$$

for all $x, y, z, t \in \mathcal{A}$, then there is a unique quartic derivation $\delta: \mathcal{A} \rightarrow \mathcal{X}$ such that

$$
\|\delta(x)-f(x)\| \leq \frac{\theta}{32-2^{p+1}}\|x\|^{p}
$$

for all $x \in \mathcal{A}$.

Proof. The proof follows from Theorem 2.5 taking

$$
\varphi(x, y, z, t):=\theta\left(\|x\|^{p}+\|y\|^{p}+\|z\|^{p}+\|t\|^{p}+\|x\|^{q_{1}}\|y\|^{q_{2}}\|z\|^{q_{3}}\|t\|^{q_{4}}\right)
$$

for all $x, y, z, t \in \mathcal{A}$.

Also, we obtain a superstability result for quartic derivations as follows:

Corollary 2.10. Let $q_{j}>0,(j=1,2,3,4)$ with $\sum_{j=1}^{4} q_{j}>4$, and $\theta$ be positive real numbers. If $f: \mathcal{A} \rightarrow \mathcal{X}$ with $f(0)=0$ is a mapping

$$
\left\|D_{f}(x, y)+f(z t)-z^{4} f(t)-f(z) t^{4}\right\| \leq \theta\left(\|x\|^{q_{1}}\|y\|^{q_{2}}\|z\|^{q_{3}}\|t\|^{q_{4}}\right)
$$

for all $x, y, z, t \in \mathcal{A}$, then $f$ is a quartic derivation.

In the following example, we show that the superstability of quartic derivations does not hold in general case.

Example 2.11. Let $x, y, z, t \in \mathcal{X}$ be fixed. We define $f: \mathcal{A} \rightarrow \mathcal{X}$ by $f(a):=a^{4} x-x a^{4}+y$ for all $a \in \mathcal{A}$,

$$
\varphi(a, b, c, d):=\left\|D_{f}(x, y)-z^{4} f(t)-f(z) t^{4}\right\|=\|y\|\left\|24+z^{4}+t^{4}\right\| .
$$

Then we have

$$
\begin{gathered}
\sum_{i=0}^{\infty} \frac{\varphi\left(2^{i} a, 0,0,0\right)}{16^{i}}=\sum_{i=0}^{\infty} \frac{\|y\|\left\|24+z^{4}+t^{4}\right\|}{16^{i}}=\frac{16}{15}\|y\|\left\|24+z^{4}+t^{4}\right\| \\
\lim _{n \rightarrow \infty} \frac{1}{16^{n}} \varphi\left(2^{n} a, 2^{n} b, 2^{n} c, 2^{n} d\right)=0
\end{gathered}
$$

for all $a, b, c, d \in \mathcal{A}$. Hence $\delta(a)=\lim _{n \rightarrow \infty} \frac{f\left(2^{n} a\right)}{16^{n}}=a^{4} x-x a^{4}$ for all $\in \mathcal{A}$. On the other hand we have

$$
\delta(a b)=(a b)^{4} x-x(a b)^{4}=a^{4} b^{4} x-x a^{4} b^{4},
$$




$$
a^{4} \delta(b)+\delta(a) b^{4}=a^{4}\left(b^{4} x-x b^{4}\right)+\left(a^{4} x-x a^{4}\right) b^{4}=a^{4} b^{4} x-x a^{4} b^{4} .
$$

Thus

$$
\delta(a b)=a^{4} \delta(b)+\delta(a) b^{4}
$$

for all $a, b \in \mathcal{A}$. Furthermore,

$\delta(2 a+b)+\delta(2 a-b)=\left[(2 a+b)^{4} x-x(2 a+b)^{4}\right]+\left[(2 a-b)^{4} x-x(2 a-b)^{4}\right]$.

On the other hand we have

$4[\delta(a+b)+\delta(a-b)]+24 \delta(a)-6 \delta(b)$

$=4\left[\left((a+b)^{4} x-x(a+b)^{4}\right)+\left((a-b)^{4} x-x(a-b)^{4}\right)\right]$

$+24\left[a^{4} x-x a^{4}\right]-6\left[b^{4} x-x b^{4}\right]$.

Then $\delta$ is quartic, that is, $D_{\delta}(a, b)=0$ for all $a, b \in \mathcal{A}$.

\section{References}

[1] Gh. Abbaspour Tabadkan and A. Rahmani, Hyers-Ulam-Rassias and UlamGavruta-Rassias stability of generalized quadratic functional equations, Advances in Applied Mathematical Analysis, Research India Publications, 4(1), pp. 31-38, (2009.

[2] S. Abbaszadeh, Intuitionistic fuzzy stability of a quadratic and quartic functional equation, Int. J. Nonlinear Anal. Appl. 1, 2, pp. 100-124, (2010).

[3] R. Badora, On approximate derivations, Math. Inequal. Appl. 9 (2006) 167173.

[4] M. Bavand Savadkouhi, M. E. Gordji, J. M. Rassias and N. Ghobadipour, Approximate ternary Jordan derivations on Banach ternary algebras, J. Math. Phys. 50, 042303 (2009), 9 pages.

[5] Bouikhalene Belaid and Elquorachi Elhoucien, Ulam-Gavruta-Rassias stability of the Pexider functional equation, Intern. J. Math. Sta. 7(Fe07), pp. 27-39, (2007).

[6] B. Bouikhalene, E. Elquorachi and J. M. Rassias, The superstability of d' Alembert's functional equation on the Heisenberg group, Appl. Math. Lett. 23, 105-109, (2010).

[7] H. X. Cao, J. R. Lv and J. M. Rassias, Superstability for generalized module left derivations and generalized module derivations on a banach module (I),Journal of Inequalities and Applications, Volume 2009, Art. ID 718020, pp. 1-10, [Doi:10.1155/2009/718020]. 
[8] H. X. Cao, J. R. Lv and J. M. Rassias, Superstability for generalized module left derivations and generalized module derivations on a banach module (II), J. Pure \& Appl. Math. JIPAM 10, Issue 2, pp. 1-8, (2009).

[9] S. Czerwik, Stability of functional equations of Ulam-Hyers-Rassias type. Hadronic Press, Palm Harbor, Florida, (2003).

[10] M. Eshaghi Gordji and M. Bavand Savadkouhi, On approximate cubic homomorphisms, Advance difference equations, Volume 2009, Article ID 618463, 11 pages ,doi:10.1155/2009/618463.

[11] M. Eshaghi Gordji, J. M. Rassias and N. Ghobadipour, Generalized HyersUlam stability of generalized (n,k)-derivations, Abstract and Applied Analysis,, pp. 1-8, (2009).

[12] M. Eshaghi Gordji, T. Karimi, S. Kaboli Gharetapeh, Approximately nJordan homomorphisms on Banach algebras, J. Ineq. Appl. Volume 2009, Article ID 870843,8 pages.

[13] M. Eshaghi Gordji, M.B. Ghaemi, S. Kaboli Gharetapeh, S. Shams, A. Ebadian, On the stability of $J^{*}$-derivations, Journal of Geometry and Physics 60 (3), pp. 454-459, (2010).

[14] M. Eshaghi Gordji, N. Ghobadipour, Stability of $(\alpha, \beta, \gamma)$-derivations on Lie $C^{*}$-algebras, International Journal of Geometric Methods in Modern Physics, Vol. 27, N 7, pp. 1-10, (2010), Doi: 10.1142/S0219887810004737.

[15] M. Eshaghi Gordji, S. Kaboli Gharetapeh, T. Karimi, E. Rashidi and M. Aghaei, Ternary Jordan derivations on $C^{*}$-ternary algebras, Journal of Computational Analysis and Applications, Vol.12, No.2, pp. 463-470, (2010).

[16] M. Eshaghi Gordji and M. S. Moslehian, A trick for investigation of approximate derivations, Math. Commun. 15, No. 1, pp. 99-105, (2010).

[17] M. Eshaghi Gordji and H. Khodaei, On the generalized Hyers-Ulam-Rassias stability of quadratic functional equations, Abstract and Applied Analysis, Volume 2009, Art. ID 923476, 1-11, Doi:10.1155/2009/923476.

[18] R. Farokhzad and S. A. R. Hosseinioun, Perturbations of Jordan higher derivations in Banach ternary algebras: An alternative fixed point approach, Internat. J. Nonlinear Anal. Appl. 1, 1, pp. 42-53, (2010).

[19] V. Faiziev and J. M. Rassias, Stability of generalized additive equations on Banach spaces and Groups", Journal of Nonlinear Functional Analysis and Differential Equations, JNFADE: 1, No. 2, 153-173, (2007).

[20] Z. Gajda, On stability of additive mappings, Internat. J. Math. Math. Sci. 14, pp. 431-434, (1991).

[21] P. Găvruta, A generalization of the Hyers-Ulam-Rassias stability of approximately additive mappings, J. Math. Anal. Appl. 184, pp. 431-436, (1994). 
[22] P. Gavruta , An answer to a question of John M. Rassias concerning the stability of Cauchy equation, in: Advances in Equations and Inequalities, in: Hadronic Math. Ser., pp. 67-71, (1999).

[23] P. Găvruta and L. Găvruta, A new method for the generalized Hyers-UlamRassias stability, Int. J. Nonlinear Anal. Appl. 1, 2, pp. 11-18, (2010).

[24] M. E. Gordji, S. Kaboli Gharetapeh, J. M. Rassias and S. Zolfaghari, Solution and Stability of a Mixed type Additive, Quadratic, and Cubic functional equation, Advances in Difference Equations, Volume 2009, Art. ID 826130, 1-17, Doi:10.1155/2009/826130.

[25] M. E. Gordji and A. Najati, Approximately $J^{*}$-homomorphisms: A fixed point approach, Journal of Geometry and Physics 60, pp. 809-814, (2010).

[26] M. E. Gordji , J. M. Rassias and M. B. Savadkouhi, Approximation of the Quadratic and Cubic functional equations in RN-spaces, European Journal of Pure and Applied Mathematics, 2, No.4, pp. 494-507, (2009.

[27] M. E. Gordji, S. Zolfaghari , J. M. Rassias and M. B. Savadkouhi, Solution and Stability of a Mixed type Cubic and Quartic functional equation in Quasi-Banach spaces, Abstr. Appl.Anal., V. 2009, Art. ID 417473, 1-14, Doi:10.1155/2009/417473.

[28] D. H. Hyers, On the stability of the linear functional equation, Proc. Natl. Acad. Sci. 27, pp. 222-224, (1941).

[29] G. Isac and Th. M. Rassias, On the Hyers-Ulam stability of additive mappings, J. Approx. Theorey 72, pp. 131-137, (1993).

[30] K. Jun, H. Kim and J. Rassias, Extended Hyers-Ulam stability for Cauchy-Jensen mappings, J. Diff. Equ. Appl. 2007, 1-15, DOI:10.1080/10236190701464590.

[31] H. Khodaei and Th. M. Rassias, Approximately generalized additive functions in several variables, Int. J. Nonlinear Anal. Appl. 1, 1, pp. 22-41, (2010).

[32] H.-M. Kim, J. M. Rassias and Y.-S. Cho, Stability Problem of Ulam for Euler-Lagrange Quadratic Mappings, Journal of Inequalities and Appl., vol., ID 10725, pp. 1-15, (2007).

[33] Z. Kominek, On a local stability of the Jensen functional equation, Demonstratio Math. 22, pp. 499-507; (1989).

[34] Y. Lee and S. Chung, Stability of an Euler-Lagrange-Rassias equation in the spaces of generalized functions, Appl. Math. Letters, doi: 10.1016/j.aml, 2007.07.022, pp.1-7.

[35] T. Miura, G. Hirasawa, S.-E. Takahasi, A perturbation of ring derivations on Banach algebras, J. Math. Anal. Appl. 319, pp. 522-530, (2006). 
[36] P. Nakmahachalasint,On the generalized Ulam-Gavruta-Rassias stability of mixed-type linear and Euler-Lagrange-Rassias functional equations, Intern. J. Math. Math. Sci. (IJMMS), Art. ID 63239, 1-10; (2007).

[37] Paisan Nakmahachalasint, Hyers-Ulam-Rassias and Ulam-Gavruta-Rassias stabilities of additive functional equation in several variables ,International Journal of Mathematics and Mathematical Sciences, Volume 2007, Art. ID 13437, 6 pages, [Doi:10.1155/2007/13437].

[38] C. Park and A. Najati, Homomorphisms and derivations in $\mathrm{C}^{*}$-algebras, $A b$ str. Appl. Anal., Article ID 80630, (2007).

[39] C. Park, Lie *-homomorphisms between Lie $\mathrm{C}^{*}$-algebras and Lie *derivations on Lie C*-algebras, J. Math. Anal. Appl. 293, pp. 419-434, (2004).

[40] W. G. Park and J. H. Bae, On a bi-quadratic functional equation and its stability Nonlinear Anal. 62, no. 4, pp. 643-654, (2005).

[41] C. Park and M. E. Gordji, Comment on Approximate ternary Jordan derivations on Banach ternary algebras [ Bavand Savadkouhi et al. J. Math. Phys. 50, 042303 (2009)], J. Math. Phys. 51, 044102 (2010) (7 pages).

[42] C. Park and A. Najati, Generalized additive functional inequalities in Banach algebras, Int. J. Nonlinear Anal. Appl. 1, 2, pp. 54-62, (2010).

[43] C. Park and J. M. Rassias, Stability of the Jensen-type functional equation in $\mathrm{C}^{*}$-algebras: A Fixed Point Approach, Abstract and Applied Analysis, Volume 2009, Art. ID 360432, 1-17, Doi:10.1155/2009/360432.

[44] C. Park and Th.M. Rassias, Isomorphisms in unital $C^{*}$-algebras, Int. J. Nonlinear Anal. Appl. 1 (2010),2, 1-10.

[45] A. Pietrzyk, Stability of the Euler-Lagrange-Rassias functional equation, Demonstratio Math. 39, 523-530, (2006).

[46] J. M. Rassias, On approximation of approximately linear mappings by linear mappings, J. Funct. Anal. 46, pp. 126-130, (1982).

[47] J. M. Rassias, On Approximation of approximately linear mappings by linear mappings, Bull. Sc. Math. 108, pp. 445-446, (1984).

[48] J. M. Rassias, On a new approximation of approximately linear mappings by linear mappings, Discuss. Math. 7, pp. 193-196, (1985).

[49] J. M. Rassias, Solution of a problem of Ulam, J. Approx. Theory. 57 (3), pp. 268-273, (1989).

[50] J. M. Rassias, On the stability of the Euler-Lagrange functional equation, Chinese J. Math. 20 (2), pp. 185-190, (1992).

[51] J. M. Rassias, Solution of a stability problem of Ulam, Discuss. Math. 12, pp. 95-103, (1992). 
[52] J. M. Rassias, On the stability of the non-linear Euler-Lagrange functional equation in real normed linear spaces, J. Math. Phys. Sci. 28, pp. 231-235, (1994).

[53] J. M. Rassias, On the stability of a multi-dimensional Cauchy type functional equation, in: Geometry, Analysis and Mechanics, World Sci. Publ., pp. 365$376,(1994)$.

[54] J. M. Rassias, Complete solution of the multi-dimensional problem of Ulam, Discuss. Math. 14, pp. 101-107, (1994).

[55] J. M. Rassias, J. Lee and H. M. Kim, Refined Hyers-Ulam stability for Jensen type mappings, Journal of the Chungcheong Mathematical Society, 22, No. 1, pp. 101-116, (2009).

[56] J. M. Rassias and M. J. Rassias, On some approximately quadratic mappings being exactly quadratic, J. Ind. Math. Soc. 69, pp. 155-160, (2002).

[57] Th. M. Rassias, The problem of S. M. Ulam for approximately multiplicative mappings, J. Math. Anal. Appl. 246 (2), pp. 352-378, (2000).

[58] Th. M. Rassias, Functional equations, Inequalities and applications, Kluwer Academic Publishers, Dordrecht, Boston and London, (2003).

[59] Th. M. Rassias, On the stability of the quadratic functional equation and its applications, Studia Univ. Babes-Bolyai Math. 43(3), pp. 89-124, (1998).

[60] Th. M. Rassias, On the stability of the linear mapping in Banach spaces, Proc. Amer.Math. Soc. 72, pp. 297-300, (1978).

[61] K. Ravi, M. Arunkumar, J.M. Rassias, Ulam stability for the orthogonally general Euler-Lagrange type functional equation, Int. J. Math. Stat. 3, pp. 36-46, (2008).

[62] P. Semrl, The functional equation of multiplicative derivation is superstable on standard operator algebras, Integral Equations Operator Theory, 18, pp. 118-122, (1994).

[63] M. A. Sibaha, B. Bouikhalene, E. Elquorachi, Ulam-Găvruta-Rassias stability for a linear functional equation, in: Euler's Issue FIDA, in: IJAMAS, vol. 7, pp. 157-167, (2007).

[64] I. M. Singer and J. Wermer, Derivations on commutative normed algebras, Math. Ann. 129, pp. 260-264, (1955).

[65] S. M. Ulam, A Collection of the Mathematical Problems, Interscience Publ., New York, (1940). 


\section{Eshaghi Gordji}

Department of Mathematics,

Semnan University,

P. O. Box 35195-363, Semnan,

Iran

e-mail: madjid.eshaghi@gmail.com

and

\section{N. Ghobadipour}

Department of Mathematics, Urmia University, Urmia,

Iran

e-mail: ghobadipour.n@gmail.com 